\title{
Pinning Group Synchronization in Complex Dynamical Networks with Different Groups of Oscillators
}

\author{
Guangming Deng ${ }^{1,2}$ \\ ${ }^{1}$ College of Science, Guilin University of Technology, Guilin 541004, China \\ ${ }^{2}$ Guangxi Key Laboratory of Spatial Information and Geomatics, Guilin 541004, China \\ Correspondence should be addressed to Guangming Deng; dgm@glut.edu.cn
}

Received 13 November 2013; Revised 19 January 2014; Accepted 20 January 2014; Published 4 March 2014

Academic Editor: Baocang Ding

Copyright (c) 2014 Guangming Deng. This is an open access article distributed under the Creative Commons Attribution License, which permits unrestricted use, distribution, and reproduction in any medium, provided the original work is properly cited.

\begin{abstract}
This paper investigates the pinning control schemes and corresponding criteria for group synchronization in a complex dynamical network with different types of chaotic oscillators. We present the linear pinning and adaptive pinning control schemes to make different groups of oscillators synchronize to their own synchronization states, respectively. The globally asymptotically stable criteria for group synchronization are derived, which indicate that the group synchronization can be realized only by pinning a part of nodes in a general network. Finally, some numerical simulations are provided to verify the theoretical results.
\end{abstract}

\section{Introduction}

Nowadays, complex networks widely exist in nature and society, such as the World Wide Web, the Internet, traffic network, communication network, power network, and social network. Over the past three decades, complex networks have received increasing attention from various fields such as biology, physics, chemistry, engineering, and information [13].

Synchronization is a kind of typical collective behaviors and basic motion in nature. As a typical network dynamics, the synchronization of complex networks has been intensively studied recently. Different synchronization phenomena have been revealed, such as global synchronization, generalized synchronization, partial synchronization, and cluster synchronization [4-11]. In particular, the group (cluster) synchronization, which means that nodes in the same group achieve the same synchronization state while nodes in different groups achieve different synchronization states, is considered to be significant in biological science, laser technology, and communication engineering $[1,2,12]$. In [13], the authors investigated the group synchronization in complex networks with two clusters of coupled dynamical systems and revealed the group synchronization motions whenever there are connections or not within a cluster. In [14], the authors investigated the group synchronization in delay-coupled dynamical networks with groups of different and identical local dynamics, respectively. In [15], we presented a complex dynamical network model with $M$ groups of different chaotic oscillators and derived some criteria to guarantee the group synchronization for a linearly or adaptively coupled network, respectively, under the "same input" condition. However, in many cases, the "same input" condition is too conservative to satisfy. If the "same input" condition cannot be satisfied, does the network achieve group synchronization? In this paper, we will propose control schemes to solve this problem.

As we known now, the real-world complex networks normally have a large number of nodes. Therefore, it is usually difficult to control a complex network by adding the controllers to all nodes. To reduce the number of the controllers, a usual approach, which is called the pinning control, is used to control part of nodes. Wang and Chen [16] and $\mathrm{Li}$ et al. [17] proposed some effective methods to pin a complex dynamical network to its equilibrium. In [18], Chen et al. proved that a single controller can pin a coupled complex network to a homogenous solution. Zhou et al. presented a simple approximate formula for estimating the detailed number of pinning nodes and the magitude of the coupling strength for a given general complex dynamical network in [19]. However, the researchers mentioned above focused on 
the global synchronization of complex dynamical networks by pinning control. In this work, we will use the pinning control schemes to realize the group synchronization in a general complex dynamical network.

In this paper, based on the model in [15], we further investigate the group synchronization in a complex network containing $M$ groups of different oscillators. Different from [15], this work mainly focuses on the pinning control schemes and the corresponding criteria to realize the group synchronization in a complex dynamical network. In particular, the network has a general structure rather than the "same input" condition.

The rest of the paper is organized as follows. In Section 2, the network model of our research and mathematical preliminaries are introduced. In Section 3, we present the linear feedback and adaptive feedback pinning schemes, respectively. The corresponding criteria for the globally asymptotically stable of the group synchronization manifold are derived. In Section 4, some numerical simulations are provided to verify the theoretical results. Finally, a brief summary is given in Section 5 .

\section{Model Description and Mathematical Preliminaries}

Let us consider a complex dynamical network with $M$ groups; each group contains a number of identical dynamical systems, but the individual dynamic system in different groups can be different. Suppose that there are $N_{I}$ nodes in $I$ th group, and the individual dynamics satisfies $\dot{x}_{i}^{I}=F_{I}\left(x_{i}^{I}\right)$. The $M$ groups network is described by

$$
\dot{x}_{i}^{I}=F_{I}\left(x_{i}^{I}\right)+c \sum_{k \neq i}^{N_{I}} a_{i k}^{I I}\left(x_{k}^{I}-x_{i}^{I}\right)+c \sum_{J \neq I}^{M} \sum_{j=1}^{N_{J}} a_{i j}^{I J}\left(x_{j}^{J}-x_{i}^{I}\right),
$$

$$
i=1,2, \ldots, N_{I}, J=1,2, \ldots, M \text {, }
$$

where $x_{i}^{I} \in R^{n_{I}}$ is state vector of the $i$ th node in the Ith group. $F_{I}$ is a smooth nonlinear function vector which describes the node's dynamics of the Ith group. $c>0$ is the coupling strength among nodes. The matrix $A_{I J}=$ $\left(a_{i j}^{I J}\right) \in R^{N_{I} \times N_{J}}$ represents the connection between $I$ th and $J$ th groups, where if node $i$ in $I$ th cluster and node $j$ in $J$ th cluster is connected, $a_{i j}^{I J}=1$; otherwise, $a_{i j}^{I J}=0$. In particular, matrix $A_{I I}$ represents the internal connection in $I$ th group, while $A_{I J}(I \neq J)$ represents the external connection between the $I$ th and $J$ th group.

Furthermore, denote the block matrix $A=\left(A_{I J}\right)$, which represents the structure of network (1). The diagonal elements of matrix $A$ satisfy $a_{i i}^{I I}=-\sum_{I=1}^{M} \sum_{j=1}^{N_{I}} a_{i j}^{I J}, i=1,2, \ldots, N_{I}$. Then $A$ is a $N \times N$ symmetric irreducible matrix with zerorow-sum, and $\operatorname{Rank}(A)=N-1$.

Symbol Description. The constant I represents $I$ th group or the set of nodes in $I$ th group. $\widetilde{I}$ represents the subset of $I$ containing the nodes with external connections; that is, $\widetilde{I}=$ $\left\{i: i \in I, \sum_{J \neq I}^{M} \sum_{j=1}^{N_{J}} a_{i j}^{I J} \neq 0\right\}$, and $I \backslash \widetilde{I}=\left\{i: i \in I, a_{i j}^{I J}=0\right.$ for $I \neq J\}$. $N_{I}$ and $l_{I}$ denote the number of nodes of set $I$ (Ith group) and $\widetilde{I}$, respectively. $N$ is the total number of nodes in network (1). $\|\cdot\|$ is the Euclidian 2-norm; that is, $\|x\|=\sqrt{x^{T} x}$ for vector $x$.

Assumption 1(A1). Suppose that there exists positive constant $\varepsilon_{k}$ such that

$$
\left\|F_{k}\left(\mathbf{z}_{1}\right)-F_{k}\left(\mathbf{z}_{2}\right)\right\| \leq \varepsilon_{k}\left\|\mathbf{z}_{1}-\mathbf{z}_{2}\right\|
$$

where $\mathbf{z}_{1}, \mathbf{z}_{2}$ are time-varying vectors.

Definition 2. The set

$$
\begin{gathered}
S=\left\{\left(x_{1}^{1}, \ldots, x_{N_{1}}^{1}, \ldots, x_{1}^{M}, \ldots, x_{N_{M}}^{M}\right): x_{i}^{I}=s_{I},\right. \\
\left.i=1, \ldots, N_{I}, I=1, \ldots, M\right\}
\end{gathered}
$$

is called group synchronous manifold of network (1).

Here, $s_{I}(t)$ is the solution of the individual dynamic system of the $I$ th group satisfying $\dot{s}_{I}(t)=F_{I}\left(s_{I}(t)\right)$. Our objective is to stable the homogenous trajectory of $x_{i}^{I}(i=$ $\left.1,2, \ldots, N_{I}\right)$ to $s_{I}(t), I=1,2, \ldots, M . s_{I}(t)$ may be an equilibrium point, a periodic orbit, an aperiodic orbit, or a chaotic orbit in the phase space.

The controlled network (1) can be rewritten as

$$
\begin{array}{r}
\dot{x}_{i}^{I}=F_{I}\left(x_{i}^{I}\right)+c \sum_{k \neq i}^{N_{I}} a_{i k}^{I I}\left(x_{k}^{I}-x_{i}^{I}\right)+c \sum_{J \neq I}^{M} \sum_{j=1}^{N_{J}} a_{i j}^{I J}\left(x_{j}^{J}-x_{i}^{I}\right)+u_{i}^{I}, \\
i=1,2, \ldots, N_{I}, J=1,2, \ldots, M .
\end{array}
$$

Define the synchronous error vectors of the $I$ th group as $e_{i}^{I}=x_{i}^{I}-s_{I}\left(i=1, \ldots, N_{I}\right)$ and $E_{I}=\left(\left(e_{1}^{I}\right)^{T}, \ldots,\left(e_{N_{I}}^{I}\right)^{T}\right)^{T}$. Then the error equations are given by

$$
\begin{gathered}
\dot{e}_{i}^{I}=F_{I}\left(x_{i}^{I}\right)-F_{I}\left(s_{I}\right)+c \sum_{k \neq i}^{N_{I}} a_{i k}^{I I} e_{k}^{I}+c \sum_{J \neq I}^{M} \sum_{j=1}^{N_{J}} a_{i j}^{I J} e_{j}^{J} \\
+c \sum_{J=1}^{M} \sum_{j=1}^{N_{I}} a_{i j}^{I J}\left(s_{J}-x_{i}^{I}\right)+u_{i}^{I}, \\
i=1,2, \ldots, N_{I}, J=1,2, \ldots, M
\end{gathered}
$$

For $i \in \tilde{I}$, there is

$$
\begin{aligned}
& \sum_{J=1}^{M} \sum_{j=1}^{N_{J}} a_{i j}^{I J}\left(s_{J}-x_{i}^{I}\right) \\
& \quad=\sum_{J=1}^{M} \sum_{j=1}^{N_{J}} a_{i j}^{I J} s_{J}-\sum_{J=1}^{M} \sum_{j=1}^{N_{I}} a_{i j}^{I J} x_{i}^{I}=\sum_{J=1}^{M} \sum_{j=1}^{N_{J}} a_{i j}^{I J} s_{J} .
\end{aligned}
$$


For $i \in \mathrm{I} \backslash \widetilde{I}$, then $a_{i j}^{I J}=0$ for $I \neq J$; there is

$$
\begin{aligned}
& \sum_{J=1}^{M} \sum_{j=1}^{N_{J}} a_{i j}^{I J}\left(s_{J}-x_{i}^{I}\right) \\
& =\sum_{k=1}^{N_{I}} a_{i k}^{I I}\left(s_{I}-x_{i}^{I}\right)+\sum_{J \neq I}^{M} \sum_{j=1}^{N_{J}} a_{i j}^{I J}\left(s_{J}-x_{i}^{I}\right) \\
& =\sum_{k=1}^{N_{I}} a_{i k}^{I I}\left(s_{I}-x_{i}^{I}\right)=\sum_{J=1}^{M} \sum_{j=1}^{N_{J}} a_{i j}^{I J}\left(s_{I}-x_{i}^{I}\right)=0 .
\end{aligned}
$$

Then the controlled error system (5) can be rewritten as

$$
\begin{gathered}
\dot{e}_{i}^{I}=F_{I}\left(x_{i}^{I}\right)-F_{I}\left(s_{I}\right)+c \sum_{k \neq i}^{N_{I}} a_{i k}^{I I} e_{k}^{I} \\
+c \sum_{J \neq I}^{M} \sum_{j=1}^{N_{J}} a_{i j}^{I J} e_{j}^{J}+c \sum_{J=1}^{M} \sum_{j=1}^{N_{I}} a_{i j}^{I J} s_{J}+u_{i}^{I}, \quad i \in \widetilde{I}, \\
\dot{e}_{i}^{I}=F_{I}\left(x_{i}^{I}\right)-F_{I}\left(s_{I}\right)+c \sum_{k \neq i}^{N_{I}} a_{i k}^{I I} e_{k}^{I}+u_{i}^{I}, \\
i \in I \backslash \widetilde{I}, I=1,2, \ldots, M .
\end{gathered}
$$

Obviously, the stability problem of group synchronous manifold $S$ of the controlled network (1) is equivalent to the stability of system (8) at zero, that is, $\lim _{t \rightarrow+\infty}\left\|e_{i}^{I}\right\|=$ $0\left(i=1, \ldots, N_{I}, I=1, \ldots, M\right)$, which implies that group synchronization is achieved.

In order to analyze the group synchronization, the following lemmas are needed.

Lemma 3 (see [20]). Suppose that $F, H$ are the $N \times N$ Hermitian matrices, and the eigenvalues of $F, H, F+H$ are $\alpha_{1} \geq \alpha_{1} \geq \cdots \geq \alpha_{N}, \beta_{1} \geq \beta_{1} \geq \cdots \geq \beta_{N}$, and $\gamma_{1} \geq \gamma_{1} \geq \cdots \geq$ $\gamma_{N}$, respectively. Then there is

$$
\alpha_{i}+\beta_{N} \leq \gamma_{i} \leq \alpha_{i}+\beta_{1}, \quad 1 \leq i \leq N .
$$

Lemma 4 (see [18]). If $G=\left(g_{i j}\right)_{N \times N}$ is an irreducible matrix with $\operatorname{Rank}(G)=N-1$, satisfying $g_{i j}=g_{j i} \geq 0$ for $i \neq j$, and $\sum_{j=1}^{N} g_{i j}=0(i=1, \ldots, N)$, then, for any constant $\varepsilon>0$, all eigenvalues of the matrix

$$
\widetilde{G}=\left(\begin{array}{cccc}
g_{11}-\varepsilon & g_{12} & \cdots & g_{1 N} \\
g_{21} & g_{22} & \cdots & a_{2 N} \\
\vdots & \vdots & \ddots & \vdots \\
g_{N 1} & g_{N 2} & \cdots & g_{N N}
\end{array}\right)
$$

are negative.

Corollary 5. Suppose that $G=\left(g_{i j}\right)_{N \times N}$ satisfies the conditions of Lemma 4. Matrix $\Delta=\operatorname{diag}\left\{\varepsilon_{1}, \ldots, \varepsilon_{l}, 0, \ldots, 0\right\}$ for $\varepsilon_{l}>0,1 \leq l<N$. Then all eigenvalues of $\widehat{G}=G-\Delta$ are negative.
Proof. If $l=1$, obviously, the conclusion is established by Lemma 4 . If $l>1$, then $\widehat{G}$ can be rewritten as $\widehat{G}=\widetilde{G}-\widetilde{\Delta}$, where $\widetilde{G}$ is in the form of (10), $\widetilde{\Delta}=\operatorname{diag}\left\{0, \varepsilon_{2}, \ldots, \varepsilon_{l}, 0, \ldots, 0\right\}$.

By Lemma 4 , all eigenvalues of $\widetilde{G}$ are negative. Denote the eigenvalues of $\widehat{G}$ and $\widetilde{G}$ as $\widehat{\lambda}_{1} \geq \widehat{\lambda}_{2} \geq \cdots \geq \widehat{\lambda}_{N}$ and $0>\widetilde{\lambda}_{1} \geq$ $\tilde{\lambda}_{2} \geq \cdots \geq \tilde{\lambda}_{N}$, respectively.

According to Lemma 3, one has

$$
\begin{array}{r}
\tilde{\lambda}_{i}-\max _{1 \leq i \leq l}\left\{\varepsilon_{i}\right\} \leq \hat{\lambda}_{i} \leq \tilde{\lambda}_{i}+0<0, \\
1 \leq i \leq N-l+1, \\
\tilde{\lambda}_{N}-\varepsilon_{i} \leq \hat{\lambda}_{i} \leq \tilde{\lambda}_{1}-\varepsilon_{i}<0, \\
N-l+1<i \leq N .
\end{array}
$$

That is, all eigenvalues of $\widehat{G}$ are negative. Thus the proof is completed.

Remark 6. From the derivation process, the conclusion of Corollary 5 is also valid when $\varepsilon_{1}, \ldots, \varepsilon_{l}$ are in any positions of the diagonal of $\Delta$; that is to say, the result holds without any dependence on the sequence of the diagonal elements of $\Delta$.

\section{Schemes and Criteria}

In this section, we present the linear feedback and adaptive feedback pinning schemes, respectively, and derive the corresponding criteria to achieve the group synchronization in network (1).

3.1. Linear Feedback Pinning Control for Group Synchronization. For network (1), we add the following linear feedback pinning controllers:

$$
u_{i}^{I}= \begin{cases}-c d_{i}^{I} e_{i}^{I}-c \sum_{J=1}^{M} \sum_{j=1}^{N_{J}} a_{i j}^{I J} s_{J}, & i \in \widetilde{I}, \\ 0, & i \in I \backslash \widetilde{I} .\end{cases}
$$

Then the error equation (8) can be rewritten as

$$
\begin{array}{r}
\dot{e}_{i}^{I}=F_{I}\left(x_{i}^{I}\right)-F_{I}\left(s_{I}\right)+c \sum_{J=1}^{M} \sum_{j=1}^{N_{J}} a_{i j}^{I J} e_{j}^{J}-c d_{i}^{I} e_{i}^{I}, \\
d_{i}^{I}>0 \quad \text { for } i \in \widetilde{I} ; \quad d_{i}^{I}=0 \quad \text { for } i \in I \backslash \widetilde{I}, \\
i=1,2, \ldots, N_{I}, I=1,2, \ldots, M .
\end{array}
$$

Theorem 7. Suppose that A1 holds. The group synchronous manifold $S$ of the controlled network (1) under the linear feedback pinning controllers (12) is globally asymptotically stable if the following inequality holds:

$$
\min _{1 \leq I \leq M}\left\{c^{-1} \varepsilon_{I}\right\}+\lambda_{\max }(A-D)<0,
$$

where $A=\left(A_{I J}\right)_{N \times N}, D=\operatorname{diag}\{\underbrace{d_{1}^{1}, \ldots, d_{l_{1}}^{1}, 0, \ldots, 0}_{N_{1}}, \ldots$, $\underbrace{\left.d_{1}^{M}, \ldots, d_{l_{M}}^{M}, 0, \ldots, 0\right\}}_{N_{M}}$ 
Proof. Consider the Lyapunov function $V(t)$ $(1 / 2) \sum_{I=1}^{M} \sum_{i=1}^{N_{I}}\left(e_{i}^{I}\right)^{T} e_{i}^{I}$.

Its time derivative along the trajectory of (13) is

$$
\begin{aligned}
\dot{V}(t)= & \sum_{I=1}^{M} \sum_{i=1}^{N_{I}}\left(e_{i}^{I}\right)^{T}\left(F_{I}\left(x_{i}^{I}\right)-F_{I}\left(s_{I}\right)\right) \\
& +c \sum_{I=1}^{M} \sum_{i=1}^{N_{I}}\left(e_{i}^{I}\right)^{T} \sum_{J=1}^{M} \sum_{j=1}^{N_{I}} a_{i j}^{I J} e_{j}^{J} \\
& -c \sum_{I=1}^{M} \sum_{i=1}^{N_{I}}\left(e_{i}^{I}\right)^{T} d_{i}^{I} e_{i}^{I} \\
\leq & \sum_{I=1}^{M} \sum_{i=1}^{N_{I}}\left\|\left(e_{i}^{I}\right)^{T}\right\| \cdot\left\|F_{I}\left(x_{i}^{I}\right)-F_{I}\left(s_{I}\right)\right\| \\
& +c \sum_{I=1}^{M} \sum_{i=1}^{N_{I}} \sum_{J=1}^{M} \sum_{j=1}^{N_{J}}\left(e_{i}^{I}\right)^{T} a_{i j}^{I J} e_{j}^{J} \\
& -c \sum_{I=1}^{M} \sum_{i=1}^{N_{I}}\left(e_{i}^{I}\right)^{T} d_{i}^{I} e_{i}^{I} \\
= & E^{T}\left(\varepsilon_{I} I_{N_{I}} \otimes I_{M}\right) E+c E^{T} A E-c E^{T} D E \\
= & c E^{T}\left(P+\left(A-\sum_{I}^{N_{I}}\left\|\left(e_{i=1}^{I}\right)^{T}\right\| \varepsilon_{I}\left\|x_{i}^{I}-s_{I}\right\|\right.\right. \\
& +c \sum_{I=1}^{M} \sum_{i=1}^{N_{I}}\left(e_{i}^{I} \sum^{I} \sum_{i=1}^{N_{I}} \sum_{J=1}^{M} d_{j}^{I} e_{j=1}^{I}\left(e_{i}^{I}\right)^{T} a_{i j}^{I J} e_{j}^{J}\right. \\
& +c \sum_{I=1}^{M} \sum_{i=1}^{N_{I}} \sum_{J=1}^{M} \sum_{j=1}^{N_{I}}\left(e_{i}^{I}\right)^{T} a_{i j}^{I J} e_{j}^{J} \\
& -c \sum_{I=1}^{M} \sum_{i=1}^{N_{I}}\left(e_{i=1}^{N_{I}} \varepsilon_{I}\right)^{T} d_{i}^{I} e_{i}^{I} e_{i}^{I} e_{i}^{I} \\
& \\
&
\end{aligned}
$$

where $P=\operatorname{diag}\{\underbrace{c^{-1} \varepsilon_{1}, \ldots, c^{-1} \varepsilon_{1}}_{N_{1}}, \ldots, \underbrace{c^{-1} \varepsilon_{M}, \ldots, c^{-1} \varepsilon_{M}}_{N_{M}}\}, E=$ $\left(\left(E_{1}\right)^{T}, \ldots,\left(E_{M}\right)^{T}\right)^{T}$.

According to the Corollary 5, $A-D$ is negative definite.

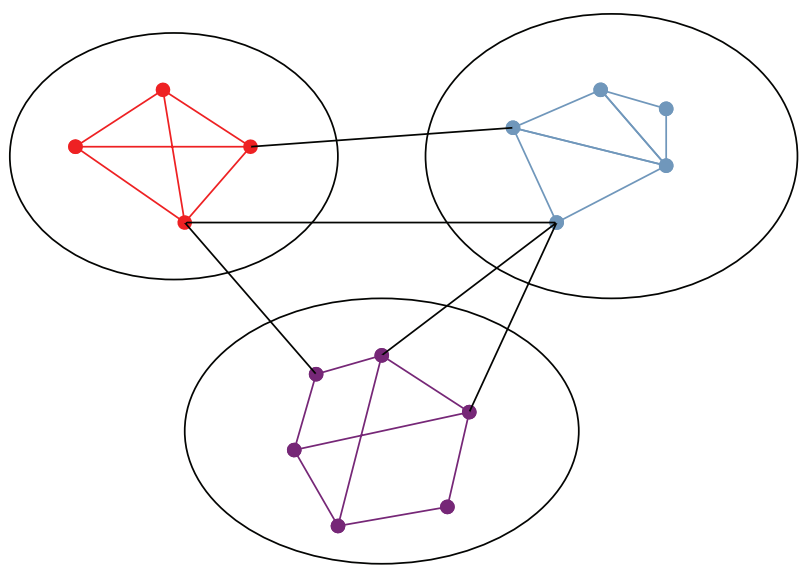

FIGURE 1: The topology connection of network (23).

Denote the eigenvalues of $A-D$ and $P+(A-D)$ as $0=$ $\lambda_{1}>\lambda_{2} \geq \cdots \geq \lambda_{N}$ and $\gamma_{1} \geq \gamma_{2} \geq \cdots \geq \gamma_{N}$, respectively. The eigenvalues of $P$ are $\beta_{j}, j=1,2, \ldots, N$; there is

$$
\min _{1 \leq I \leq M}\left\{c^{-1} \varepsilon_{I}\right\} \leq \beta_{j} \leq \max _{1 \leq I \leq M}\left\{c^{-1} \varepsilon_{I}\right\} .
$$

According to Lemma 3, one has

$$
\begin{array}{r}
\lambda_{N}+\beta_{j} \leq \gamma_{j} \leq \lambda_{1}+\beta_{j} \leq \lambda_{1}+\max _{1 \leq I \leq M}\left\{c^{-1} \varepsilon_{I}\right\} \\
=\lambda_{\max }(A-D)+\max _{1 \leq I \leq M}\left\{c^{-1} \varepsilon_{I}\right\}<0, \\
j=1,2, \ldots, N .
\end{array}
$$

That is, $P+(A-D)$ is negative definite. Then one can obtain $\lim _{t \rightarrow+\infty}\left\|e_{i}^{I}\right\|=0\left(i=1, \ldots, N_{I}, I=1, \ldots, M\right)$, which means that the group synchronous manifold $S$ is globally asymptotically stable. Thus the proof is completed.

Remark 8. From Theorem 7, one can obtain the sufficient condition (14) by means of adjusting the coupling strength $c$. Therefore, the group synchronization can be guaranteed.

Remark 9. In this linear feedback pinning scheme, one only needs to pin a few nodes which have external connections; then the network achieves the group synchronization with large enough coupling strength. In particular, it is only relevant to network connectivity, without any limitation to the network structure. For a cluster network, in general, the connection within a cluster is more than the external connection between clusters. When the network has a cluster property, the higher the clustering, the better the control effect. Since the network with higher clustering means that the number of nodes with external connection is less, thus the less nodes are needed to be pinned. Therefore, the higher the degree of clustering, the more obvious the advantages.

3.2. Adaptive Feedback Pinning Control for Group Synchronization. In order to avoid estimating the feedback control gain, in this section, we present an adaptive feedback pining scheme to realize the group synchronization in network (1). 


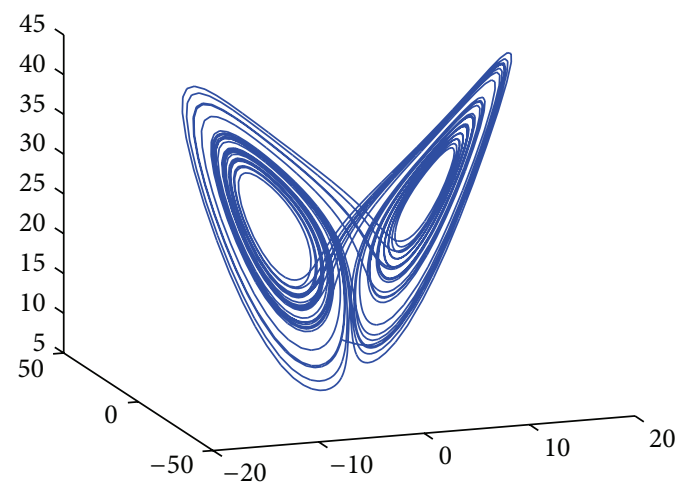

— Lorenz attractor

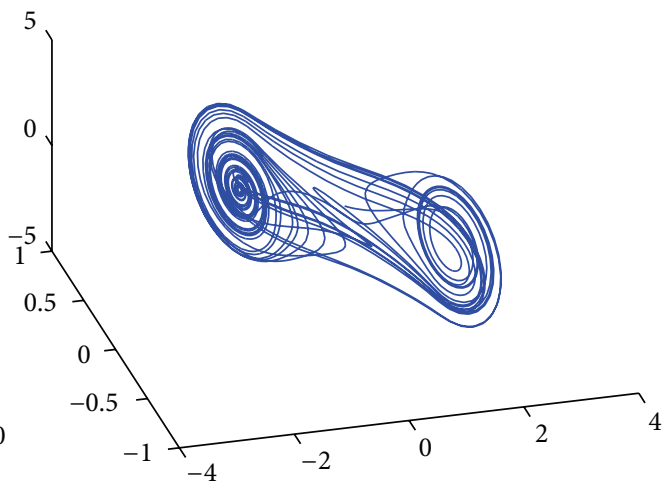

— Chua attractor

(a)

(b)

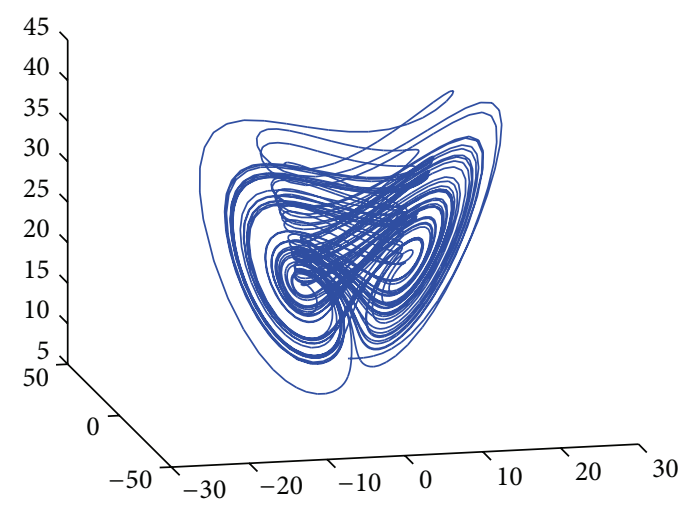

— Chen attractor

(c)

FIGURE 2: The chaotic attractors of Lorenz (a), Chua (b), and Chen (c).

For network (1), we add the adaptive feedback pinning controllers as follows:

$$
u_{i}^{I}=\left\{\begin{array}{l}
-c d_{i}^{I}(t) e_{i}^{I}-c \sum_{J=1}^{M} \sum_{j=1}^{N_{J}} a_{i j}^{I J} s_{J}, \\
\dot{d}_{i}^{I}(t)=c d\left(e_{i}^{I}\right)^{T} e_{i}^{I}, \quad d>0, \text { for } i \in \widetilde{I} \\
0, \\
\text { for } i \in I \backslash \widetilde{I} .
\end{array}\right.
$$

Then the error equation (8) can be rewritten as

$$
\begin{aligned}
& \dot{e}_{i}^{I}=F_{I}\left(x_{i}^{I}\right)-F_{I}\left(s_{I}\right)+c \sum_{J=1}^{M} \sum_{j=1}^{N_{J}} a_{i j}^{I J} e_{j}^{J}-c d_{i}^{I} e_{i}^{I}, \\
& \dot{d}_{i}^{I}=c d\left(e_{i}^{I}\right)^{T} e_{i}^{I} \quad \text { for } i \in \widetilde{I} ; \quad d_{i}^{I}=0 \quad \text { for } i \in I \backslash \widetilde{I}, \\
& \quad i=1,2, \ldots, N_{I}, I=1,2, \ldots, M .
\end{aligned}
$$

Theorem 10. Suppose that A1 holds. The group synchronous manifold $S$ of the controlled network (1) under the adaptive feedback pinning controllers (18) is globally asymptotically stable if the following inequality holds:

$$
\min _{1 \leq I \leq M}\left\{c^{-1} \varepsilon_{I}\right\}+\lambda_{\max }(A-\widetilde{D})<0
$$

where $A=\left(A_{I J}\right)_{N \times N}, \widetilde{D}=\operatorname{diag}\{\underbrace{d, \ldots, d}_{l_{1}}, \underbrace{0, \ldots, 0}_{N_{1}-l_{1}}, \ldots$, $\underbrace{d, \ldots, d}_{l_{M}}, \underbrace{0, \ldots, 0}_{N_{M}-l_{M}}\}$.

Proof. Consider the Lyapunov function

$$
W(t)=\frac{1}{2} \sum_{I=1}^{M} \sum_{i=1}^{N_{I}}\left(e_{i}^{I}\right)^{T} e_{i}^{I}+\frac{1}{2 d} \sum_{I=1}^{M} \sum_{i=1}^{N_{I}}\left(d_{i}^{I}-d\right)^{2}
$$




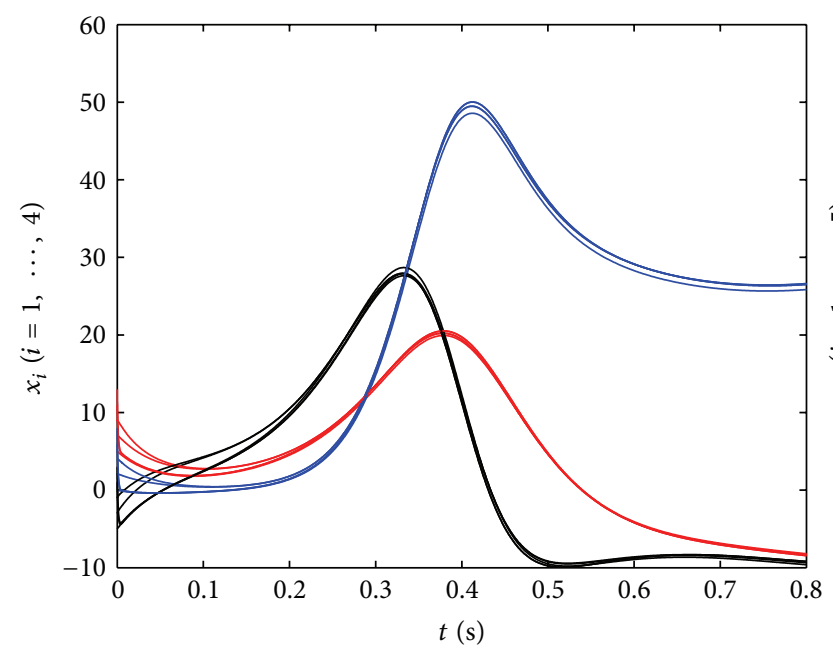

(a)

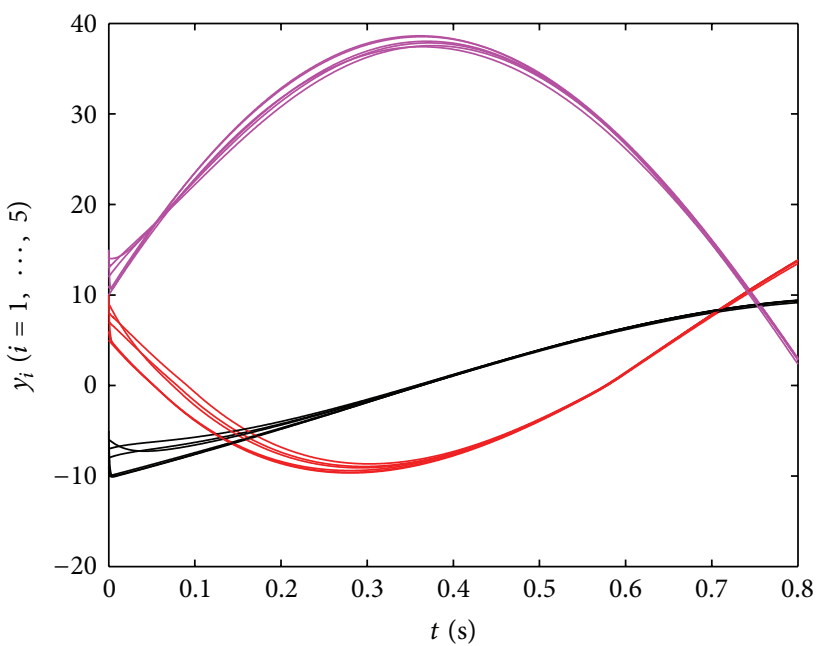

(b)

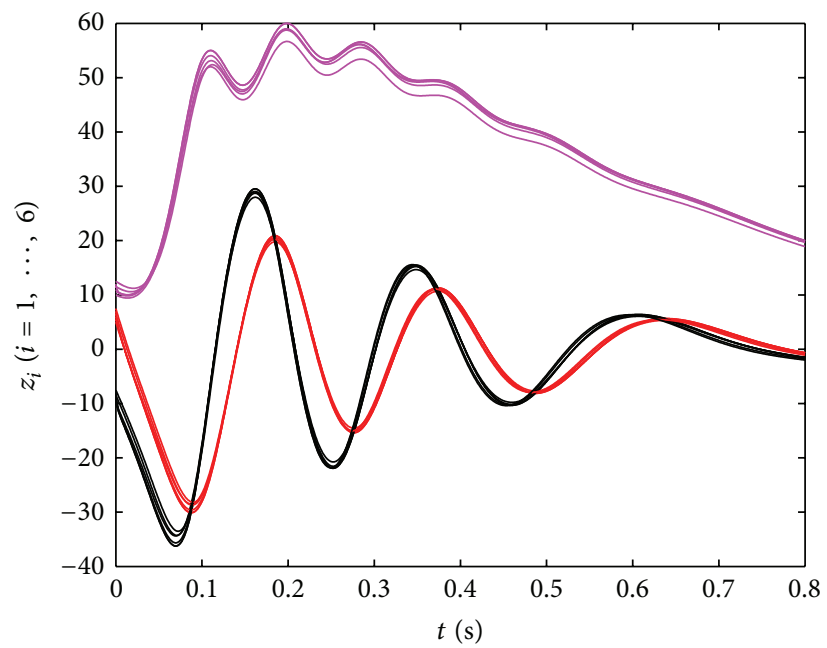

(c)

FIGURE 3: Group synchronization of network (23) with linear feedback pinning controllers (12). (a) The synchrony states $x_{i}(1 \leq i \leq 4)$ of Lorenz oscillators in $x$-group; (b) the synchrony states $y_{i}(1 \leq i \leq 5)$ of Chua oscillators in $y$-group; (c) the synchrony states $z_{i}(1 \leq i \leq 6)$ of Chen oscillators in $z$-group.

Its time derivative along the trajectory of (19) is

$$
\begin{aligned}
\dot{W}= & \sum_{I=1}^{M} \sum_{i=1}^{N_{I}}\left(e_{i}^{I}\right)^{T} \dot{e}_{i}^{I}+\frac{1}{d} \sum_{I=1}^{M} \sum_{i=1}^{N_{I}}\left(d_{i}^{I}-d\right) \dot{d}_{i}^{I} \\
= & \sum_{I=1}^{M} \sum_{i=1}^{N_{I}}\left(e_{i}^{I}\right)^{T}\left(F_{I}\left(x_{i}^{I}\right)-F_{I}\left(s_{I}\right)\right) \\
& +c \sum_{I=1}^{M} \sum_{i=1}^{N_{I}}\left(e_{i}^{I}\right)^{T} \sum_{J=1}^{M} \sum_{j=1}^{N_{J}} a_{i j}^{I J} e_{j}^{J}-c \sum_{I=1}^{M} \sum_{i=1}^{N_{I}}\left(e_{i}^{I}\right)^{T} d_{i}^{I} \mathrm{e}_{i}^{I} \\
& +c \sum_{I=1}^{M} \sum_{i=1}^{N_{I}}\left(d_{i}^{I}-d\right)\left(e_{i}^{I}\right)^{T} e_{i}^{I}
\end{aligned}
$$

$$
\begin{aligned}
\leq & \sum_{I=1}^{M} \sum_{i=1}^{N_{I}}\left(e_{i}^{I}\right)^{T} \varepsilon_{i} e_{i}^{I}+c \sum_{I=1}^{M} \sum_{i=1}^{N_{I}} \sum_{J=1}^{M} \sum_{j=1}^{N_{J}}\left(e_{i}^{I}\right)^{T} a_{i j}^{I J} e_{j}^{J} \\
& -c \sum_{I=1}^{M} \sum_{i=1}^{N_{I}}\left(e_{i}^{I}\right)^{T} \mathrm{~d} e_{i}^{I} \\
= & c E^{T}(P+(A-\widetilde{D})) E .
\end{aligned}
$$

The rest is similar to the proof of Theorem 7; one has $\lim _{t \rightarrow+\infty}\left\|e_{i}^{I}\right\|=0\left(i=1, \ldots, N_{I}, I=1, \ldots, M\right)$, which means that the group synchronous manifold $S$ is globally asymptotically stable. Thus the proof is completed. 


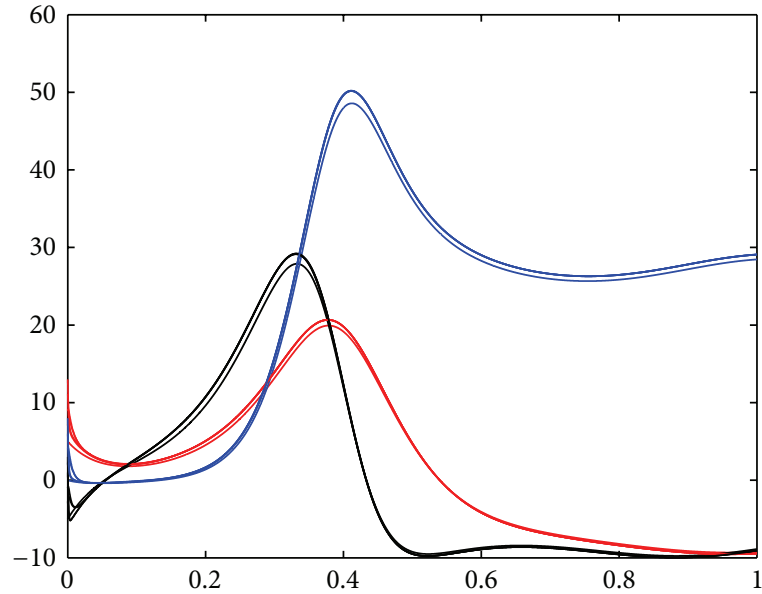

(a)

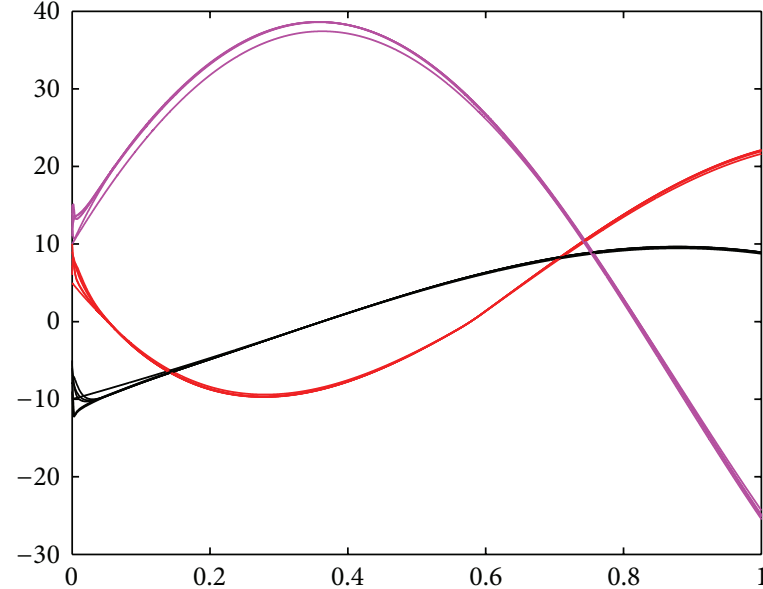

(b)

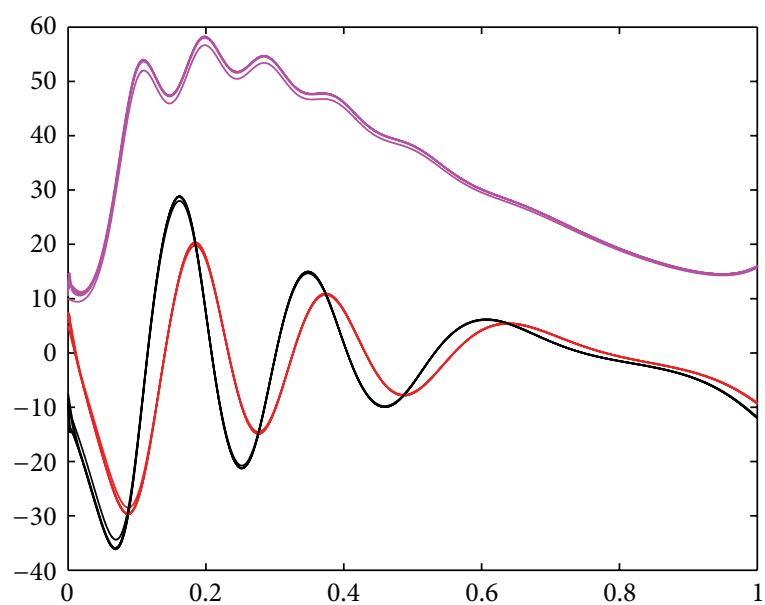

(c)

FIGURE 4: Group synchronization of network (23) with adaptive feedback pinning controllers (18). (a) The synchrony states $x_{i}(1 \leq i \leq 4)$ of Lorenz oscillators in $x$-group; (b) the synchrony states $y_{i}(1 \leq i \leq 5)$ of Chua oscillators in $y$-group; (c) the synchrony states $z_{i}(1 \leq i \leq 6)$ of Chen oscillators in $z$-group.

Remark 11. In Theorem 10, the adaptive constant $d$ can be large enough such that inequality (20) holds. Therefore, the group synchronization can be easily guaranteed.

\section{Numerical Simulations}

In this section, an illustrative example is provided to verify the above theoretical analysis.

We consider a network (see Figure 1) which consists of three groups with 4 Lorenz chaotic oscillators [20] as the $x$ group, 5 Chua oscillators [21] as the $y$-group, and 6 Chen oscillators [22] as the $z$-group, respectively. The Lorenz, Chua, and Chen chaotic attractors are displayed in Figure 2.

The network as an example of the controlled network (1) is described by

$$
\dot{x}_{i}=F_{1}\left(x_{i}\right)+c \sum_{j=1}^{4} a_{i j} x_{j}+c \sum_{j=1}^{5} b_{i j}\left(y_{j}-x_{i}\right)
$$

$$
+c \sum_{j=1}^{6} h_{i j}\left(z_{j}-x_{i}\right)+u_{i}^{1}, \quad 1 \leq i \leq 4,
$$

$$
\begin{array}{r}
\dot{y}_{i}=F_{2}\left(y_{i}\right)+c \sum_{j=1}^{5} \bar{a}_{i j} y_{j}+c \sum_{j=1}^{4} \bar{b}_{i j}\left(x_{j}-y_{i}\right) \\
+c \sum_{j=1}^{6} \bar{h}_{i j}\left(z_{j}-y_{i}\right)+u_{i}^{2}, \quad 1 \leq i \leq 5,
\end{array}
$$

$$
\begin{aligned}
\dot{z}_{i}=F_{3}\left(z_{i}\right)+c \sum_{j=1}^{6} \widetilde{a}_{i j} z_{j} \\
\quad+c \sum_{j=1}^{4} \widetilde{b}_{i j}\left(x_{j}-z_{i}\right)+c \sum_{j=1}^{5} \widetilde{h}_{i j}\left(y_{j}-z_{i}\right)+u_{i}^{3},
\end{aligned}
$$

$1 \leq i \leq 6$, 
where $F_{1}\left(x_{i}\right)=\left(10\left(x_{i 2}-x_{i 1}\right), 28 x_{i 1}-x_{i 2}-x_{i 1} x_{i 3}, x_{i 1} x_{i 2}-\right.$ $\left.8 / 3 x_{i 3}\right)^{T} ; F_{2}\left(y_{i}\right)=\left(10\left(y_{i 2}-y_{i 1}\right)-f\left(y_{i 1}\right), y_{i 1}-y_{i 2}+\right.$ $\left.y_{i 3},-14.7 y_{i 2}\right)^{T}, f(v)=m_{0} v+0.5\left(m_{1}-m_{0}\right)(|x+1|-|x-1|), m_{0}=$ $-0.68, m_{1}=-1.27 ; F_{3}\left(z_{i}\right)=\left(35\left(z_{i 2}-z_{i 1}\right),-7 z_{i 1}+28 z_{i 2}-\right.$ $\left.z_{i 1} z_{i 3}, z_{i 1} z_{i 2}-8 / 3 z_{i 3}\right)^{T}$. The controllers $u_{i}^{I}$ are taken as $(12)$ and (18), respectively.

In the simulation, we take the initial values $x_{i}(0)=(-5+$ $0.5 i, 0.5 i, 5+0.5 i)^{T}(1 \leq i \leq 4), y_{i}(0)=(-5+0.5 i, 0.5 i, 5+$ $0.5 i, 10+0.5 i)^{T}(1 \leq i \leq 5), z_{i}(0)=(-5+0.5 i, 0.5 i, 5+$ $0.5 i)^{T}(1 \leq i \leq 6)$, and $d(0)=1$.

Figures 3 and 4 display the numerical simulation results of network (23) under the linear feedback and adaptive feedback pinning control, respectively. They both show that a set of nodes belonging to each group synchronizes to the same behavior; that is, the group synchronization has been achieved.

Remark 12. By the above theoretical analysis and instance, it is concluded that the pinning control strategy can be applied to large networks with tens of thousands of nodes based on the accurate network topology. In fact, this control strategy depends on the structure information rather than the size of the network.

\section{Conclusions}

In this paper, we have investigated the group synchronization in a complex dynamical network consisting of different groups of coupled chaotic oscillators. The linear feedback and adaptive feedback pinning schemes are presented, respectively. The corresponding sufficient conditions are derived to ensure each group synchronizes to the same states. Our study shows that the global asymptotic stability of the group synchronous manifold can be guaranteed by controlling a few nodes which have external connections. For the large networks with obvious cluster property, our pinning control schemes can save much cost in practical.

\section{Conflict of Interests}

The author declares that there is no conflict of interests regarding the publication of this paper.

\section{Acknowledgments}

This work was jointly supported by the National Natural Science Foundation (no. 61164020), the National Social Science Foundation (no. 13BTJ009), the Natural Science Foundation of Guangxi (0991244, 2011 GXNSFA018147), Guangxi Key Laboratory of Spatial Information and Geomatics (nos. 1103108-24, 1207115-27).

\section{References}

[1] M. E. J. Newman, "The structure and function of complex networks," SIAM Review, vol. 45, no. 2, pp. 167-256, 2003.
[2] S. Boccaletti, V. Latora, Y. Moreno, M. Chavez, and D. U. Hwang, "Complex networks: structure and dynamics," Physics Reports, vol. 424, no. 4-5, pp. 175-308, 2006.

[3] A. Arenas, A. Díaz-Guilera, J. Kurths, Y. Moreno, and C. Zhou, "Synchronization in complex networks," Physics Reports, vol. 469, no. 3, pp. 93-153, 2008.

[4] Q. Zhang, J. Lu, J. Lu, and C. K. Tse, "Adaptive feedback synchronization of a general complex dynamical network with delayed nodes," IEEE Transactions on Circuits and Systems II, vol. 55, no. 2, pp. 183-187, 2008.

[5] A. Y. Pogromsky, "A partial synchronization theorem," Chaos, vol. 18, Article ID 037107, 2008.

[6] X. Wu, W. X. Zheng, and J. Zhou, "Generalized outer synchronization between complex dynamical networks," Chaos, vol. 19, Article ID 013109, 2009.

[7] H. Liu, J. Chen, J. Lu, and M. Cao, "Generalized synchronization in complex dynamical networks via adaptive couplings," Physica A, vol. 389, no. 8, pp. 1759-1770, 2010.

[8] W. Yu, J. Cao, G. Chen, J. Lü, J. Han, and W. Wei, "Local synchronization of a complex network model," IEEE Transactions on Systems, Man, and Cybernetics B, vol. 39, no. 1, pp. 230-241, 2009.

[9] V. Belykh, I. Belykh, and E. Mosekilde, "Cluster synchronization modes in an ensemble of coupled chaotic oscillators," Physical Review E, vol. 63, no. 3, pp. 362161-362164, 2001.

[10] V. Belykh, G. Osipov, V. Petrov, J. A. K. Suykens, and J. Vandewalle, "Cluster synchronization in oscillatory networks," Chaos, vol. 18, Article ID 037106, 2008.

[11] Z. Jia and G. Deng, "Mathematical model and cluster synchronization for a complex dynamical network with two types of chaotic oscillators," Journal of Applied Mathematics, vol. 2012, Article ID 595360, 12 pages, 2012.

[12] K. Kaneko, "Relevance of dynamic clustering to biological networks," Physica D, vol. 75, no. 1-3, pp. 55-73, 1994.

[13] F. Sorrentino and E. Ott, "Network synchronization of groups," Physical Review E, vol. 76, no. 5, Article ID 056114, 2007.

[14] T. Dahms, J. Lehnert, and E. Scholl, "Cluster and group synchronization in delay-coupled networks," Physical Review E, vol. 86, Article ID 016202, 2012.

[15] Z. Jia, X. Fu, G. Deng, and K. Li, "Group synchronization in complex dynamical networks with different types of oscillators and adaptive coupling schemes," Communications in Nonlinear Science and Numerical Simulation, vol. 18, no. 10, pp. 2752-2760, 2013.

[16] X. Wang and G. Chen, "Pinning control of scale-free dynamical networks," Physica A, vol. 310, no. 3-4, pp. 521-531, 2002.

[17] X. Li, X. Wang, and G. Chen, "Pinning a complex dynamical network to its equilibrium," IEEE Transactions on Circuits and Systems I, vol. 51, no. 10, pp. 2074-2087, 2004.

[18] T. Chen, X. Liu, and W. Lu, "Pinning complex networks by a single controller," IEEE Transactions on Circuits and Systems I, vol. 54, no. 6, pp. 1317-1326, 2007.

[19] J. Zhou, J. Lu, and J. Lü, "Pinning adaptive synchronization of a general complex dynamical network," Automatica, vol. 44, no. 4, pp. 996-1003, 2008.

[20] E. N. Lorenz, "Determinstic non-periods flows," Journal of the Atmospheric Sciences, vol. 20, pp. 130-141, 1963.

[21] J. A. K. Suykens, A. Huang, and L. O. Chua, "A family of n-scroll attractors from a generalized Chuas circuit," Archiv Ekektron Uberttragungstech, vol. 51, no. 3, pp. 131-138, 1997. 
[22] G. Chen and T. Ueta, "Yet another chaotic attractor," International Journal of Bifurcation and Chaos in Applied Sciences and Engineering, vol. 9, no. 7, pp. 1465-1466, 1999. 


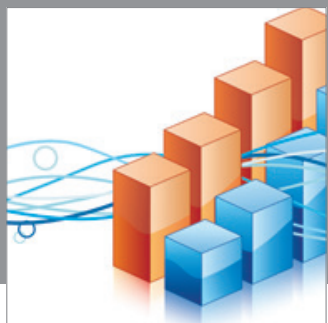

Advances in

Operations Research

mansans

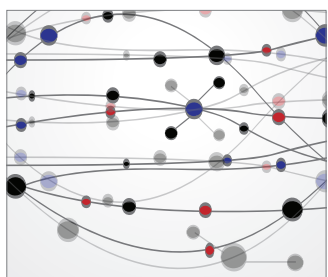

The Scientific World Journal
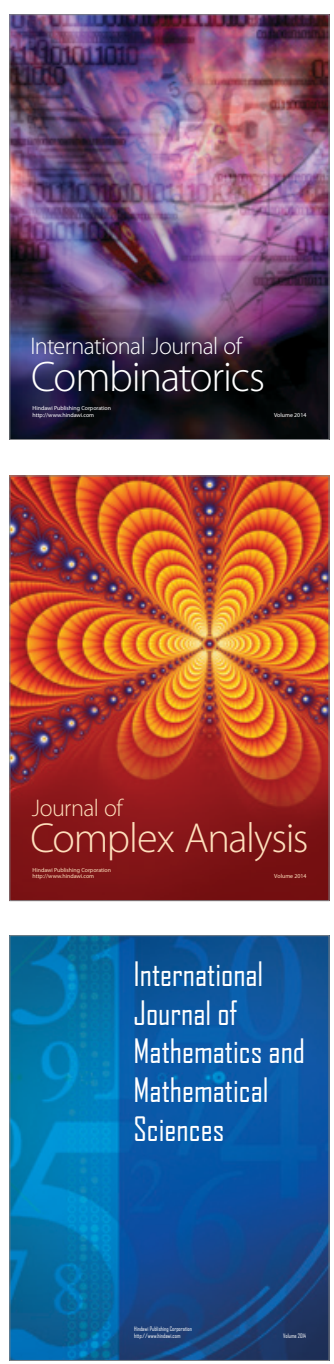
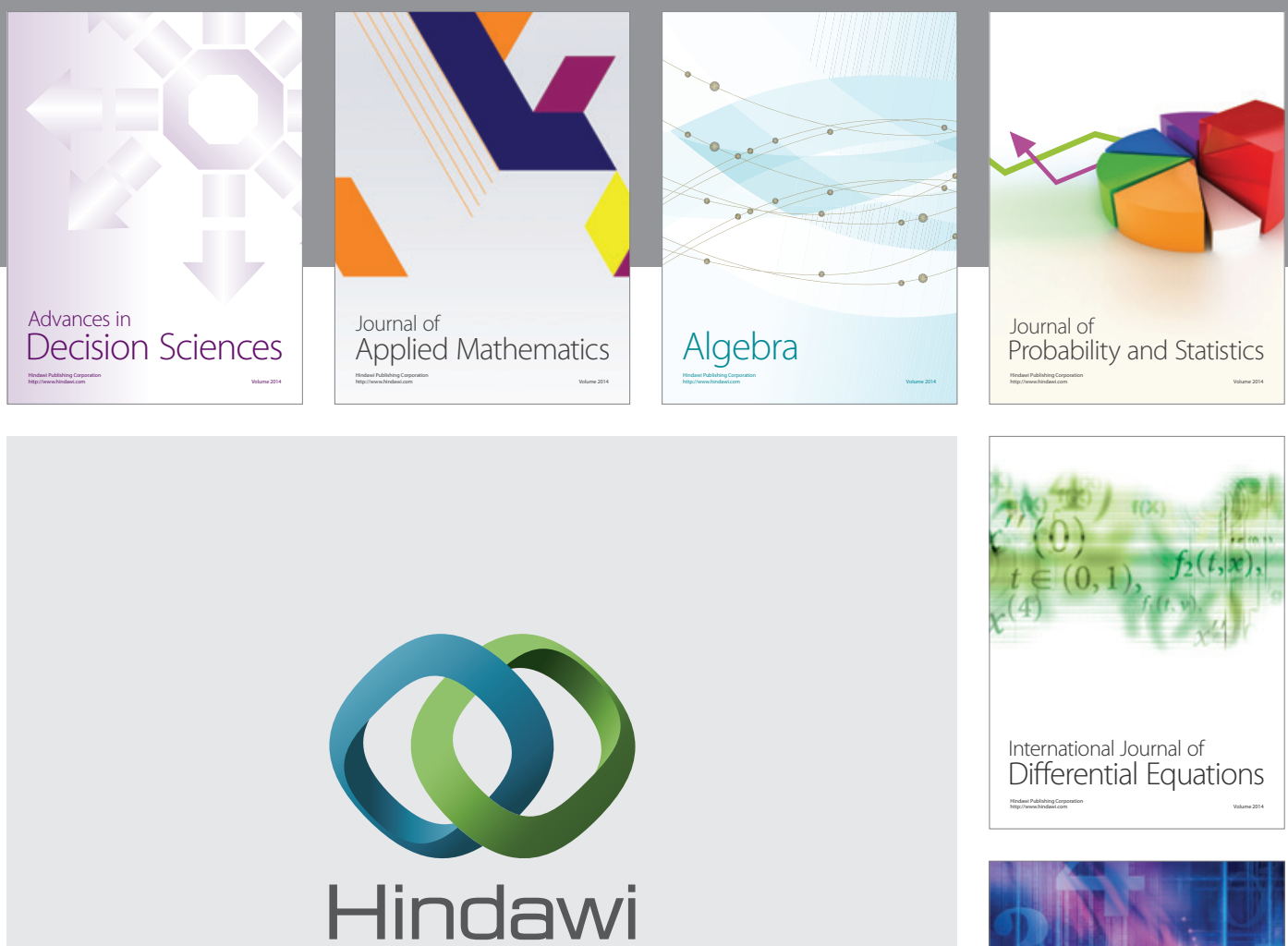

Submit your manuscripts at http://www.hindawi.com
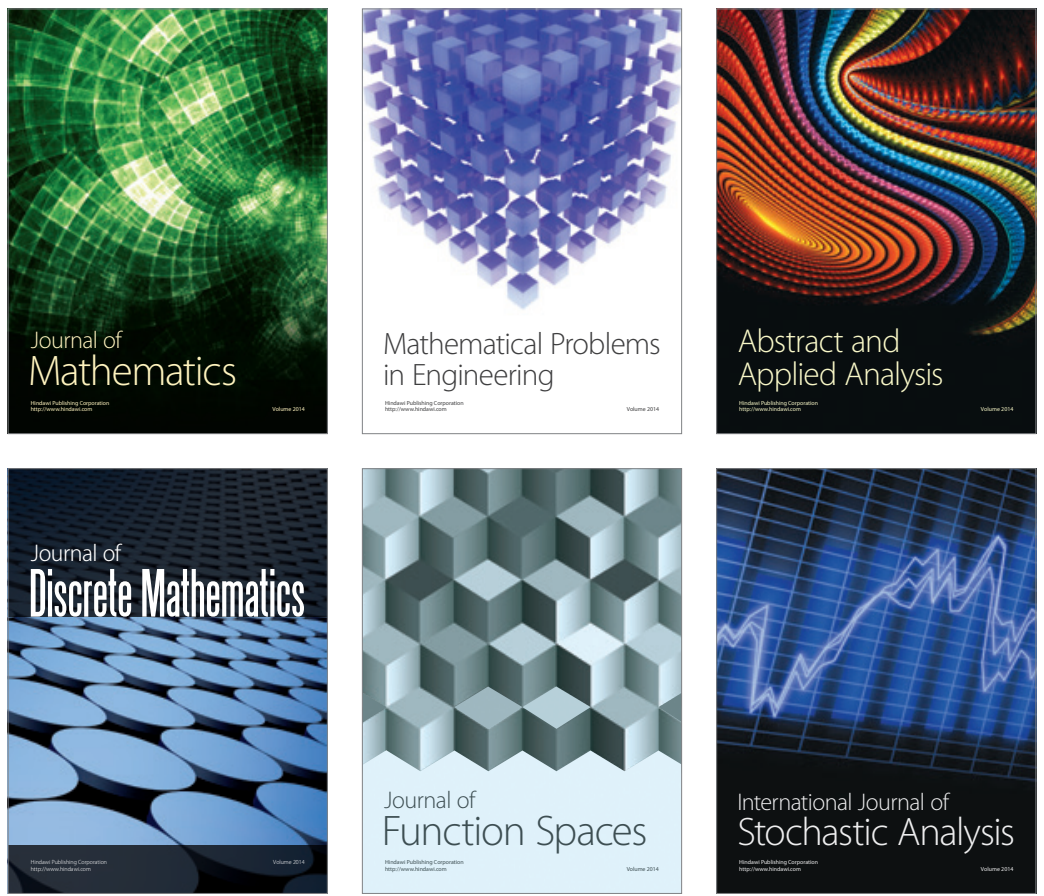

Journal of

Function Spaces

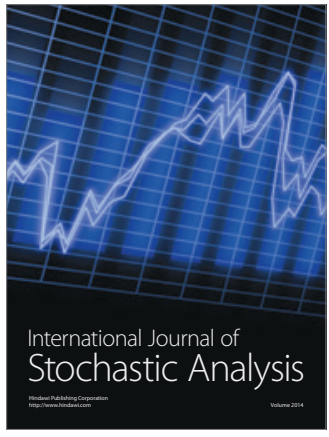

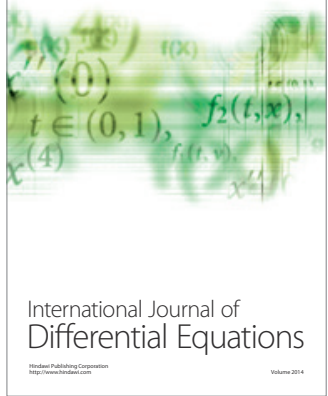
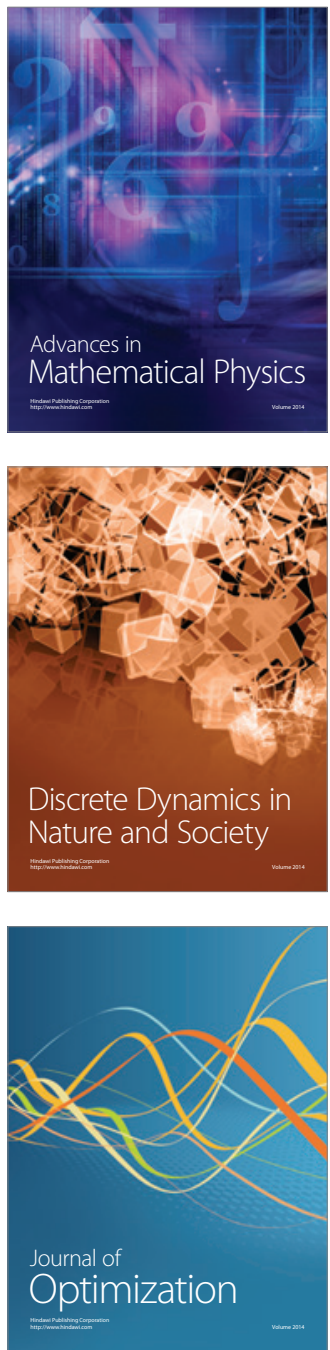